\title{
Liquid-Based Cytology Features of Papillary Squamotransitional Cell Carcinoma of the Uterine Cervix
}

\author{
Yangkyu Lee ${ }^{*}$, Younghwa Choi ${ }^{*}$, Kiryang Lee ${ }^{1}$, Youngeun Lee ${ }^{1}$, Hyojin Kim, \\ Ji-Young Choe ${ }^{2}$, Hye Seung Lee, Yong Beom Kim ${ }^{3}$, Haeryoung Kim ${ }^{1}$ \\ Department of Pathology, Seoul National University Bundang Hospital, Seoul National University College of Medicine, Seongnam; \\ ${ }^{1}$ Department of Pathology, Seoul National University Hospital, Seoul National University College of Medicine, Seoul; \\ 2Department of Pathology, Hallym University Sacred Heart Hospital, Hallym University College of Medicine, Anyang; \\ ${ }^{3}$ Department of Obstetrics and Gynecology, Seoul National University Bundang Hospital, Seoul National University College of Medicine, Seongnam, Korea
}

Papillary squamotransitional cell carcinoma (PSTCC) of the uterine cervix is an uncommon variant of cervical squamous cell carcinoma (SCC). ${ }^{1}$ Here we describe the SurePath liquid-based cytology (LBC) findings of a case of PSTCC which showed a combination of cytological features of low-grade papillary urothelial carcinoma (LGPUC) and SCC.

\section{CASE REPORT}

A 77-year-old previously healthy woman presented with bloody vaginal discharge at the outpatient clinic. A colposcopy revealed a $2.5 \mathrm{~cm}$-sized papillary mass (Fig. 1), and a punch biopsy was performed. After the possibility of a PSTCC or papillary SCC was suggested on the biopsy, she underwent a total abdominal hysterectomy with bilateral salpingo-oophorectomy. Two years later, she returned to the clinic complaining of vaginal discharge. A 6$\mathrm{mm}$-sized enhancing nodular lesion was noted at the right vaginal stump on pelvic computed tomography, and a cervicovaginal smear and punch biopsy was taken to confirm recurrence. However, further treatment of the vaginal lesion was not performed, as imaging incidentally revealed a pancreatic tail neoplasm with peritoneal seeding. The patient was subsequently referred to hospice care.

Received: May 9, 2019 Revised: May 28, 2019

Accepted: June 5, 2019

Corresponding Author: Haeryoung Kim, MD, PhD

Department of Pathology, Seoul National University College of Medicine, Basic Science

Building \#101, 103 Daehak-ro, Jongno-gu, Seoul 03080, Korea

Tel: +82-2-740-8322, Fax: +82-2-765-5600, E-mail: haeryoung.kim@snu.ac.kr

*Yangkyu Lee and Younghwa Choi contributed equally to this work.
At low-power magnification, the LBC preparation was highly cellular, showing a few small hyperchromatic crowded groups (HCGs) in a background of abundant scattered cells (Fig. 2A). At higher magnification, the HCGs were 3-dimensional irregularlyshaped papillary fragments containing fibrovascular cores. The atypical cells lining the papillary clusters were relatively uniformly distributed with mild nuclear overlapping (Fig. 2B). The nuclei were smaller than those of parabasal cells, and were round-to-oval with smooth nuclear membranes. Nucleoli were small and almost inconspicuous. Mitotic figures were only occasionally seen. Similarlooking atypical cells were individually scattered in the background (Fig. 2B, C). Some cells were spindled with long and tapered cytoplasmic processes (Fig. 2C). Altogether, most of the neoplastic cells demonstrated the cytological features of LGPUC. There were also a few interspersed cells with increased nuclearto-cytoplasmic ratio, hyperchromasia and nuclear membrane irregularity, suggestive of high-grade squamous intraepithelial lesion (HSIL) (Fig. 2D). A small cluster of dyskeratotic cells was seen (Fig. 2E, F). Tumor diathesis was not identified.

The biopsied tissue demonstrated papillary excrescences containing fibrovascular cores (Fig. 3A). The lining epithelium showed hybrid features of LGPUC and SCC (Fig. 3B, C). The LGPUClike component was seen towards the basal layers and showed mild cytological atypia. Towards the surface of the papillary stuctures, there was evidence of keratinization and high-grade cytologic atypia. Koilocytosis was focally seen (Fig. 3D). Although stromal invasion was not observed in the biopsied tissue, the subsequent hysterectomy specimen demonstrated residual tumor with stromal invasion and vascular invasion in the parametrial vessels. Immunohistochemistry revealed diffuse nuclear p63 expression, and the 
tumor cells were cytokeratin 7 (CK7)-positive and CK20-negative. A human papillomavirus (HPV) test (LG AdvanSure GenoBlot Assay, LG Life Sciences, Seoul, Korea) detected "other type" HPV. The histologic findings of the recurrent tumor were similar to

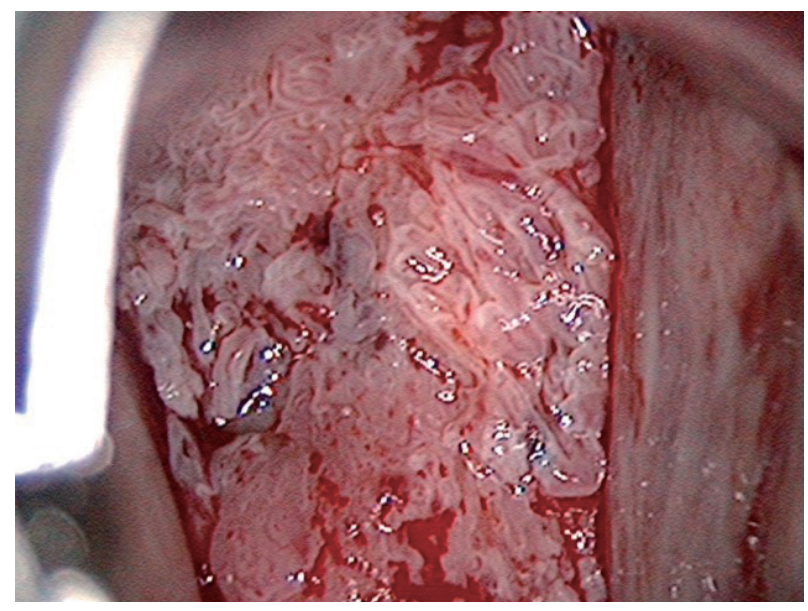

Fig. 1. Colposcopy reveals a friable papillary mass in the cervical canal. that of the initial tumor.

\section{Ethics statement}

This case study was approved by the Institutional Review Board of Seoul National University Bundang Hospital (IRB approval number B-1710-427-702) and informed consent was waived.

\section{DISCUSSION}

PSTCC was first reported in 1986 by Randall et al. ${ }^{1}$ as a variant of SCC showing varying degrees of urothelial-like differentiation. Due to the predominantly exophytic nature of these tumors, invasion may not be detected on small biopsies or cervicovaginal cytology. However, despite the surface papillary growth pattern, PSTCCs have been frequently associated with deep invasion, local recurrence and distant metastasis. ${ }^{1-3}$

While the clinicopathological features of PSTCC have been discussed in several case reports, the cytological findings of PSTCC have only been described in two reports: one study described the
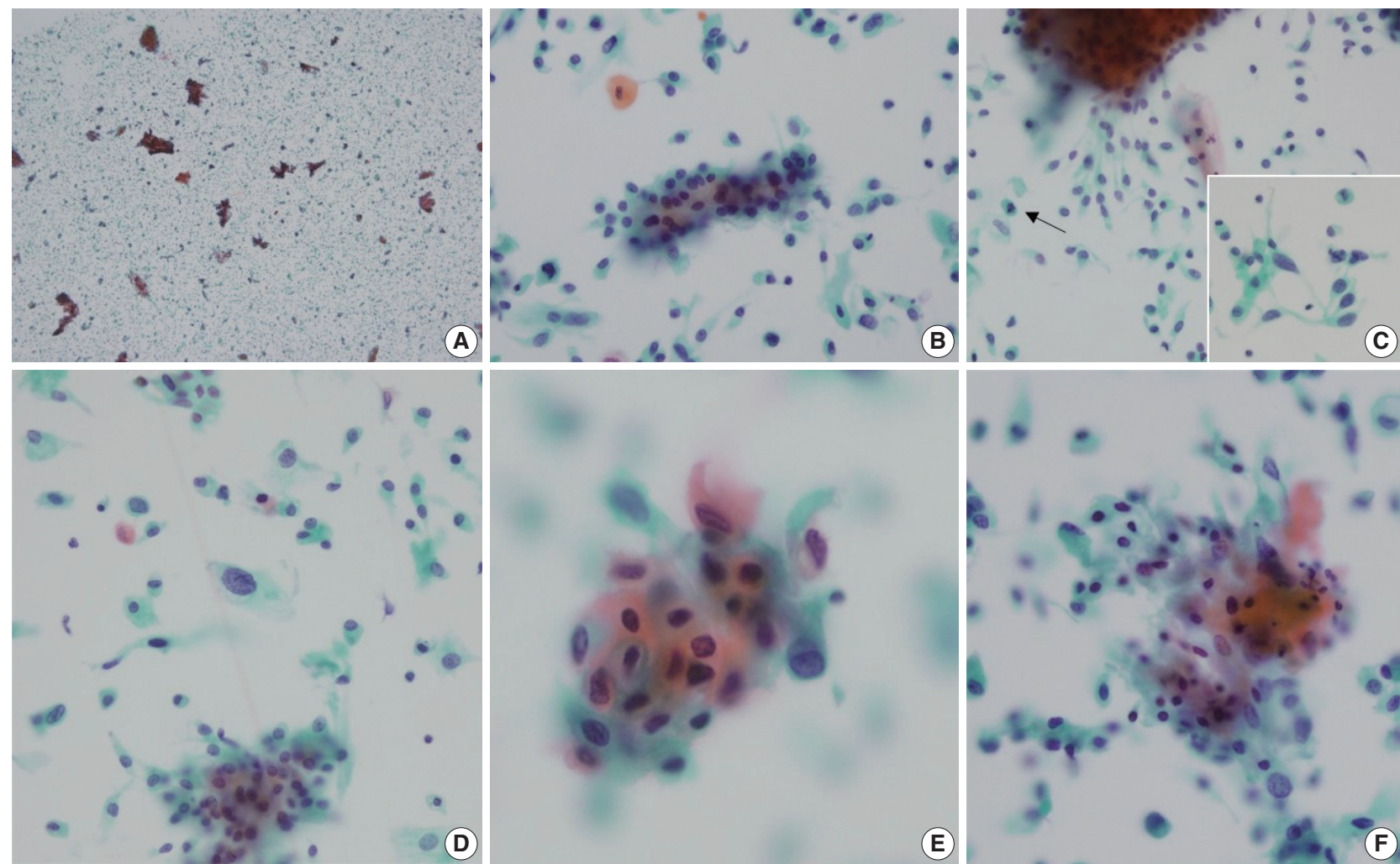

(E)

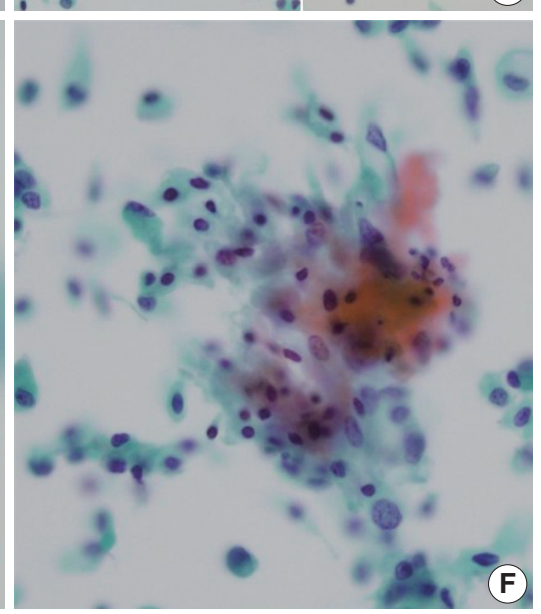

Fig. 2. Liquid-based cytology findings. (A) Low-power magnification shows some scattered hyperchromatic crowded groups and abundant individually scattered cells. (B) A papillary cluster (center) is lined by small neoplastic cells with round-to-oval nuclei and minimal nuclear atypia. (C) The scattered neoplastic cells demonstrate similar features, with eccentrically located, round-to-oval nuclei. Long cytoplasmic processes are seen in some neoplastic cells ("cercariform" cells) at the edge of papillary fragments (C, inset). A mitotic figure is seen (arrow). (D) A high-grade squamous intraepithelial lesion cell with coarse chromatin and irregular nuclear membrane (center) is seen among the scattered low-grade urothelial carcinoma-like cells. (E, F) Dyskeratotic cells and focal koilocytotic atypia are occasionally seen. 

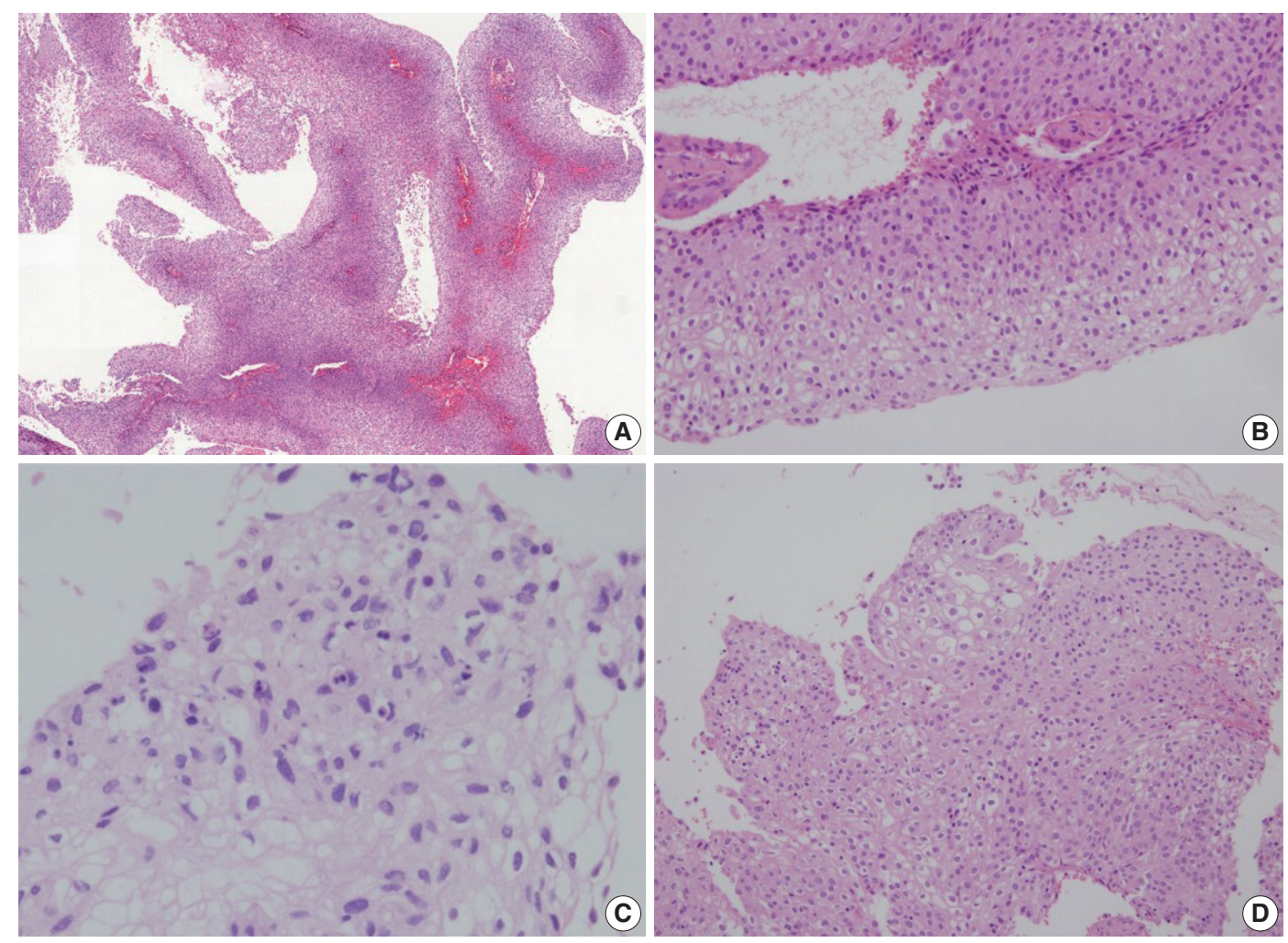

Fig. 3. Histological findings. (A) Low-power view demonstrates papillary structures with fibrovascular cores. (B) The papillae are mostly lined by urothelial-like cells. (C, D) Focal areas of keratinization (C) and koilocytotic atypia (D) are seen in the surfaces of the papillae.

LBC features of six cases, ${ }^{4}$ and the other one is a single case report describing the conventional smear features. ${ }^{5}$ The LBC features of PSTCC in this case could be summarized as follows: (1) a moderate-to-high cellularity, (2) HCGs characterized by 3-dimensional papillary fragments with branching architecture, (3) urothelial-like features, and (4) loosely dispersed hyperchromatic parabasal-like cells in the background. ${ }^{4,5}$

The differential diagnosis of PSTCC includes a spectrum of lesions ranging from reactive to neoplastic, including papillary immature metaplasia and HSIL with papillary configuration. ${ }^{6,7}$ Immature metaplastic and repair cells are immature parabasal-like cells with cytoplasmic projections, 2-dimensional sheets and fine chromatin, while PSTCCs frequently demonstrate 3-dimensional HCG with papillary architecture and hyperchromatic nuclei. Although papillary architecture may be seen in HSILs, the papillary fragments of PSTCC have been described to show more complex arborizing patterns. ${ }^{4}$ In addition, the presence of horizontally aligned cells on the surface of papillary structures—similar to that seen in LGPUC in urine cytology — has been shown to be a characteristic cytological feature of PSTCC. ${ }^{4,8}$

Despite the urothelial-like features, PSTCCs have been suggested to be metaplastic variants of SCC. The $\mathrm{CK} 7+/ \mathrm{CK} 20$ profile of PSTCC (including this case) is identical to that of müllerian epithelium. ${ }^{3}$ Moreover, similarly to SCC, HPV and allelic losses at $3 \mathrm{p}$ have been implicated in the pathogenesis of PSTCCs, while chromosome 9 alterations (common in bladder cancer) were not detected. ${ }^{3,9,10}$

\section{ORCID}

Yangkyu Lee: https://orcid.org/0000-0002-6008-7713

Younghwa Choi: https://orcid.org/0000-0002-1425-9422

Kiryang Lee: https://orcid.org/0000-0003-3769-001X

Youngeun Lee: https://orcid.org/0000-0002-0000-1615

Hyojin Kim: https://orcid.org/0000-0001-9201-8328

Ji-Young Choe: https://orcid.org/0000-0001-7967-8487

Hye Seung Lee: https://orcid.org/0000-0002-1667-7986

Yong Beom Kim: https://orcid.org/0000-0003-1196-369X

Haeryoung Kim: https://orcid.org/0000-0002-4205-9081

\section{Author Contributions}

Conceptualization: HK (Haeryoung Kim).

Data curation: YL (Yangkyu Lee), YC.

Investigation: YL (Yangkyu Lee), KL, YL (Youngeun Lee). 
Methodology: YL (Yangkyu Lee), HK (Haeryoung Kim).

Resources: HK (Hyojin Kim), JYC, HSL, YBK, HK (Haeryoung Kim).

Supervision: HK (Haeryoung Kim).

Visualization: YL (Yangkyu Lee), HK (Haeryoung Kim).

Writing—original draft: YL (Yangkyu Lee), YC, KL.

Writing—review \& editing: YL (Yangkyu Lee), KL, HK (Haeryoung Kim).

\section{Conflicts of Interest}

The authors declare that they have no potential conflicts of interest.

\section{Funding}

No funding to declare.

\section{REFERENCES}

1. Randall ME, Andersen WA, Mills SE, Kim JA. Papillary squamous cell carcinoma of the uterine cervix: a clinicopathologic study of nine cases. Int J Gynecol Pathol 1986; 5: 1-10.

2. Al-Nafussi AI, Al-Yusif R. Papillary squamotransitional cell carcinoma of the uterine cervix: an advanced stage disease despite superficial location: report of two cases and review of the literature. Eur J Gynaecol Oncol 1998; 19: 455-7.

3. Koenig C, Turnicky RP, Kankam CF, Tavassoli FA. Papillary squamotransitional cell carcinoma of the cervix: a report of 32 cases.
Am J Surg Pathol 1997; 21: 915-21.

4. Ng WK. Thin-layer (liquid-based) cytologic findings of papillary squamotransitional cell carcinoma of the cervix. Review of cases over a 4-year period with emphasis on potential diagnostic pitfalls. Acta Cytol 2003; 47: 141-8.

5. Vesoulis Z, Erhardt CA. Cytologic diagnosis of vaginal papillary squamotransitional cell carcinoma. A case report. Acta Cytol 2001; 45: 465-9.

6. Kokka F, Verma M, Singh N, Faruqi A, Yoon J, Reynolds K. Papillary squamotransitional cell carcinoma of the uterine cervix: report of three cases and review of the literature. Pathology 2006; 38: 584-6.

7. Kim HS, Seon MI, Kim YJ, Kim HS. Cytologic features of papillary immature metplasia of uterine cervix. Korean J Cytopathol 2002; 13: 21-7.

8. Chung YR, Won JK, Park IA, et al. Cytomorphological characteristics of low-grade papillary urothelial carcinoma for differential diagnosis from benign papillary urothelial lesions: logistic regression analysis in SurePath(TM) liquid-based voided urine cytology. Cytopathology 2016; 27: 83-90.

9. Fujimoto T, Sakuragi N, Shimizu M, et al. Papillary squamous cell carcinoma of the uterine cervix: a report of two cases with human papillomavirus 16 DNA. Acta Obstet Gynecol Scand 2002; 81: 176-8.

10. Maitra A, Wistuba, II, Gibbons D, Gazdar AF, Albores-Saavedra J Allelic losses at chromosome $3 p$ are seen in human papilloma virus 16 associated transitional cell carcinoma of the cervix. Gynecol Oncol 1999; 74: 361-8 